\title{
POSITIVE ONE-RELATOR GROUPS
}

\author{
BY \\ GILBERT BAUMSLAG $\left({ }^{1}\right)$ \\ Dedicated to the memory of A. I. Mal'cev.
}

\begin{abstract}
A group $G$ which can be defined by a single relation in which there are no negative exponents is residually solvable. If $G$ is also torsion-free then it is locally indicable and hence its integral group ring has no zero divisors.
\end{abstract}

\section{Introduction.}

1.1. If $X$ is a class of groups, then $R X$ denotes the class of those groups which are residually in $X$. We recall that $G \in{ }_{R} X$ if for each $g \in G(g \neq 1)$ there exists a normal subgroup $N$ of $G$ such that $g \notin N$ and $G / N \in X$.

Inspired by the work of F. W. Levi [13], W. Magnus [15], A. I. Mal'cev [18], [19] and P. Hall [7], [8], K. W. Gruenberg [6] initiated in 1958 a systematic investigation of residual properties. In the intervening years a great deal of additional work has been done (see e.g., the survey article by W. Magnus [16]). This paper is devoted to the proof of three new theorems about groups with a single defining relator which relate residual properties to the "form" of the relator. As such, our results may be viewed as part of the continuing investigation of the properties of one-relator groups which stem from the nature of the defining relator (see [2] and the references cited there, B. B. Newman [21] and B. Baumslag [1]).

1.2. As the title suggests we shall be concerned here mainly with positive onerelator groups. By this we mean a one-relator group

$$
G=\left(x_{1}, x_{2}, \ldots, x_{n} ; r\right)
$$

whose defining relator

$$
r=x_{i_{1}}^{e_{1}} x_{i_{2}}^{e_{2}} \cdots x_{i_{m}}^{e_{m}} \quad\left(e_{j}= \pm 1, i_{j} \in\{1, \ldots, n\}\right)
$$

is a positive word, i.e. $e_{j}=+1$ for $j=1,2, \ldots, m$. Among the simplest one-relator groups are the fundamental groups $G_{k}$ of nonorientable two-dimensional surfaces (see e.g. [22]):

$$
G_{k}=\left(a_{1}, a_{2}, \ldots, a_{k} ; a_{1}^{2} a_{2}^{2} \cdots a_{k}^{2}\right) \quad(k=1,2, \ldots) .
$$

Received by the editors May 10, 1970.

AMS 1970 subject classifications. Primary 20F05.

Key words and phrases. Positive one-relator group, group ring, zero divisor, residually finite, poly-residually-torsion-free-nilpotent, generalized free product, locally indicable.

( ${ }^{1}$ Support from N.S.F. is gratefully acknowledged.

Copyright (c) 1971, American Mathematical Society 
A structurally much more complicated positive one-relator group is $\left({ }^{2}\right)$ the nonhopfian group (see [5])

$$
\left(a, b ; a^{-1}(a b)^{2} a(a b)^{3}\right) .
$$

Our first concern here is to show that, despite this structural vagary, all positive one-relator groups share an uncomplicated residual property.

THEOREM 1. Every positive one-relator group is residually solvable.

Of course, not every one-relator group is residually solvable. For example, the group

$$
G=\left(a, b ; a=\left[a, b^{-1} a b\right]\right)
$$

is not residually solvable since $a \neq 1$ (by the Freiheitssatz [17]) and every term of the derived series of $G$ contains $a$.

1.3. The other main concern of this paper is with the residual nilpotence of positive one-relator groups. The best we can do here is to prove

THEOREM 2. Every positive one-relator group $G$ has an invariant series

$$
1=G_{0} \leqq G_{1} \leqq \cdots \leqq G_{k}=G \quad(k<\infty)
$$

such that

(i) $G_{1}$ is free;

(ii) $G_{2} / G_{1}$ is the direct sum of cyclic groups of order $n$, where $n$ is the maximum of the orders of the elements of finite order in $G$, and a free abelian group;

(iii) $G_{i+1} / G_{i}$ is residually-torsion-free-nilpotent for $i=2,3, \ldots, k-1$.

It follows from Theorem 2 and the residual nilpotence of free groups (see [15]) that we have

COROLlary 1. A positive one-relator group is poly-residually-nilpotent.

In particular if the positive one-relator group $G$ has no elements of finite order then we have a slightly stronger version of Corollary 1 , viz.

COROLlaRY 2. A torsion-free positive one-relator group is poly-residuallytorsion-free-nilpotent.

It follows immediately from Corollary 2 that torsion-free positive one-relator groups are locally indicable in the sense of G. Higman [9] and hence

COROLLARY 3. The integral group ring of a torsion-free positive one-relator group has no zero divisors.

Thus Theorem 2 may be considered as a first step towards proving that the integral group ring of a torsion-free one-relator group is an integral domain.

Actually the proof of Theorem 2 yields also the

COROLlaRY 4. Positive one-relator groups are poly-residually-finite.

(2) This fact was pointed out to me by Graham Higman. 
Corollary 4 suggests that all one-relator groups are poly-residually-finite. But this is false as the group $G$ given by (1) shows (see 5.3).

1.4. Finally in $\$ 6$ we prove a variation of Corollary 2 :

THEOREM 3. The generalized free product of two residually-torsion-free-nilpotent groups amalgamating a cyclic subgroup is meta-residually-torsion-free-nilpotent, i.e., an extension of one residually-torsion-free-nilpotent group by another.

By allying Theorem 3 with a theorem of I. P. Hughes [10] we deduce the following result about one-relator groups.

COROLLARY 3.1. The integral group ring of a generalized free product of two free groups amalgamating a cyclic subgroup can be embedded in a division ring.

1.5. The arrangement of the rest of this paper is as follows. In $\$ 2$ we recall the basic breakdown of one-relator groups which is crucial in any study of one-relator groups. In $\S 3$ we prepare the ground for the proof of Theorem 1 which takes up $\$ 4$. In $\S 5$ we prove Theorem 2 . Finally in $\S 6$ we prove Theorem 3.

\section{Preliminaries concerning one-relator groups.}

2.1. The least normal subgroup of a group $G$ containing the subset $Y$ of $G$ will be denoted by $\operatorname{gp}_{G}(Y)$.

2.2. We recall some notation and results from W. Magnus' theory of groups with a single defining relation (see [17]). Thus let

$$
G=(a, b, \ldots, c ; r)
$$

be a group with a single defining relator $r$. We suppose $r$ is cyclically reduced and that each of the generators appears in $r$. In addition, we assume that $a$ occurs in $r$ with exponent sum zero. Put

$$
N=\operatorname{gp}_{G}(b, \ldots, c)
$$

and write $r$ in functional form as $r=r(a, b, \ldots, c)$. Then $G / N$ is infinite cyclic. Putting

$$
b_{i}=a^{-i} b a^{i}, \ldots, \quad c_{j}=a^{-j} c a^{j} \quad(i, \ldots, j=0, \pm 1, \ldots)
$$

we see that $N$ is generated by these elements $\ldots, b_{i}, \ldots, c_{j}, \ldots$ It follows that we can rewrite $r$ in the form

$$
r_{0}=r_{0}\left(b_{m(b)}, \ldots, b_{M(b)}, \ldots, c_{m(c)}, \ldots, c_{M(c)}\right),
$$

where $r_{0}$ is obtained from $r$ by moving all occurrences of $a$ over to the left and compensating by adding the appropriate subscripts onto the relevant generator that is bypassed by $a$. Here $m(b)$ is the smallest $b$-subscript occurring while $M(b)$ is the largest, etc. Clearly the length of $r_{0}$ is definitely less than the length of $r$-this fact is essential to most proofs about one-relator groups. We shall sometimes refer to $r_{0}$ as "the basic relator in $N$ ". 
For each integer $k$, put

$$
N_{k}=\operatorname{gp}\left(b_{m(b)+k}, \ldots, b_{M(b)+k}, \ldots, c_{m(c)+k}, \ldots, c_{M(c)+k}\right) .
$$

Then $N_{k}$ is (completely) defined by the single relator

$$
r_{0}\left(b_{m(b)+k}, \ldots, b_{M(b)+k}, \ldots, c_{m(c)+k}, \ldots, c_{M(c)+k}\right) .
$$

Moreover if

$$
H_{k}=\operatorname{gp}\left(b_{m(b)+k+1}, \ldots, b_{M(b)+k}, \ldots, c_{m(c)+k+1}, \ldots, c_{M(c)+k}\right)
$$

and if

$$
N_{i, j}=\operatorname{gp}\left(N_{i}, \ldots, N_{j}\right) \quad(i \leqq j)
$$

then $N_{i, j+1}$ is the generalized free product of $N_{i, j}$ and $N_{j+1}$ amalgamating $H_{j}$ :

$$
N_{i, j+1}=\left\{N_{i, j} * N_{j+1} ; H_{j}\right\}
$$

Similarly,

$$
N_{i-1, j}=\left\{N_{i-1} * N_{i, j} ; H_{i-1}\right\} .
$$

These facts will be useful in the sequel.

\section{Preparations for the proof of Theorem 1.}

3.1. There are two aspects of the proof of Theorem 1 which should be pointed out in order to make it more palatable. The first involves the verification that under suitable circumstances, the generalized free product

$$
P=\{A * B ; H \stackrel{\varphi}{=} K\}
$$

of two residually solvable groups $A$ and $B$ amalgamating $H$ with $K$ according to the isomorphism $\varphi$ is again residually solvable. The second, which is related to the first through an induction argument, concerns the proof that, for certain subgroups $C$ of $B$,

$$
\bigcap_{i=1}^{\infty} C \delta_{i} P=C \text { whenever } \bigcap_{i=1}^{\infty} C \delta_{i} B=C,
$$

where $\delta_{i} G$ denotes the $i$ th derived group of $G(i=1,2, \ldots)$.

Here we shall formulate conditions under which results of this type hold and then during the course of the proof of Theorem 1 verify that these conditions are satisfied.

3.2. Throughout this subsection let $A$ and $B$ be residually solvable groups, let $H \leqq A, K \leqq B$ and let $\varphi$ be an isomorphism taking $H$ onto $K$. A family $\left(A_{\lambda} \mid \lambda \in \Lambda\right)$ of normal subgroups of $A$ is termed a solvable filtration of $A$ if $A / A_{\lambda}$ is solvable for every $\lambda \in \Lambda$ and $\bigcap_{\lambda \in \Lambda} A_{\lambda}=1$. We shall say that $H$ is solvably separable in $A$ if

$$
\bigcap_{i=1}^{\infty} H \delta_{i} A=H .
$$


Of course, if $\bigcap_{\lambda \in \Lambda} H A_{\lambda}=H$, then $H$ is solvably separable in $A$. A solvable filtration $\left(A_{\lambda} \mid \lambda \in \Lambda\right)$ is termed an H-filtration if $\bigcap_{\lambda \in \Lambda} H A_{\lambda}=H$. Two equally indexed filtrations $\left(A_{\lambda} \mid \lambda \in \Lambda\right)$ and $\left(B_{\lambda} \mid \lambda \in \Lambda\right)$ of $A$ and $B$ respectively are termed $(H, K, \varphi)$ compatible if

$$
\left.\left(A_{\lambda} \cap H\right) \varphi=B_{\lambda} \cap K \quad \text { (for all } \lambda \in \Lambda\right) .
$$

Under these circumstances $\varphi$ induces for each $\lambda \in \Lambda$ an isomorphism $\varphi_{\lambda}$ from $H A_{\lambda} / A_{\lambda}$ to $K B_{\lambda} / B_{\lambda}$.

The first fact we need follows easily from the argument used to prove Proposition 1 in [2].

Proposition 1. Let $\left(A_{\lambda} \mid \lambda \in \Lambda\right),\left(B_{\lambda} \mid \lambda \in \Lambda\right)$ be solvable, $(H, K, \varphi)$-compatible filtrations of the residually solvable groups $A$ and $B$ respectively. Suppose $\left(A_{\lambda} \mid \lambda \in \Lambda\right)$ is an H-filtration of $A$ and $\left(B_{\lambda} \mid \lambda \in \Lambda\right)$ is a K-filtration of $B$. If

$$
\left\{A \mid A_{\lambda} * B / B_{\lambda} ; H A_{\lambda} / A_{\lambda} \stackrel{\varphi_{\lambda}}{=} K B_{\lambda} / B_{\lambda}\right\}
$$

is residually solvable for every $\lambda \in \Lambda$ then so is

$$
P=\{A * B ; H \stackrel{\varphi}{=} K\}
$$

We shall also need

Proposition 2. Let $S$ be a subgroup of the residually solvable group $G$. If $S$ is solvably separable in $G$ and $T$ is residually solvable then both $S$ and $S * T$ are solvably separable in $G * T$.

The proof of Proposition 2 depends on the following lemma.

LemMA 3.1. A free factor $C$ of a residually solvable group $A$ is solvably separable in $A$.

Proof. Suppose that $A=B * C$. We have to prove that if $a \in A, a \notin C$, then for a suitable choice of $l, a \notin C \delta_{l} A$. Now

$$
a=x_{1} x_{2} \cdots x_{n} \quad\left(x_{i} \in B \cup C\right)
$$

where successive factors $x_{i}, x_{i+1}$ do not belong to the same subgroup. Since $a \notin C$ either $n>1$ or $x_{1} \in B\left(x_{1} \neq 1\right)$. Choose $k \geqq 1$ so that $x_{i} \notin \delta_{k} X_{i}(i=1, \ldots, n)$ where $X_{i}$ is the appropriate free factor containing $x_{i}$. Let $\eta$ be the natural homomorphism of $A$ onto

$$
\bar{A}=\bar{B} * \bar{C} \quad\left(\bar{B}=B / \delta_{k} B, \bar{C}=C / \delta_{k} C\right) .
$$

Then $a \eta \notin \bar{C}$. Thus we may restrict our attention to the case where $B$ and $C$ are actually solvable of derived length at most $k$.

Let $\mu$ be the natural homomorphism of $A$ onto $B \times C$. Then the kernel $K$ of $\mu$ is free since $K \cap A=1=K \cap B$ (see e.g. [20, p. 492]). Now $a=b c u(u \in K)$. If $b \neq 1$, then $a \notin C \delta_{k} A$. So assume $a=c u$. Notice that as $a \notin C, u \neq 1$. Now $K$ is free and 
hence $K$ is residually solvable (F. W. Levi [13]). So we can find $m$ so that $u \notin \delta_{m} K$. Then $a \notin C \delta_{m} K$. For if $a=c^{\prime} u^{\prime}\left(c^{\prime} \in C, u^{\prime} \in \delta_{m} K\right)$ then, on noting that $K \cap C=1$ and remembering that $a=c u$, we find $c=c^{\prime}, u=u^{\prime} \in \delta_{m} K$, a contradiction. But $\delta_{k+m} A \leqq \delta_{m} K$. So, putting $l=k+m$, the proof of Lemma 3.1 is complete.

We come now to the proof of Proposition 2. In the first instance the proof of the fact that $S$ is solvably separable in $H=G * T$ follows easily from the proof of Lemma 3.1. Thus we shall concentrate here on the other part of Proposition 2, i.e., we shall prove $S * T$ is solvably separable in $H$.

Again it follows easily from the proof of Lemma 3.1 that we may assume without loss of generality that both $G$ and $T$ are solvable of derived length at most $k$. Let $K$ be the kernel of the natural homomorphism of $H$ onto $G \times T$. Suppose $a \in H, a \notin S * T$. Then $a$ is uniquely of the form

$$
a=g t u \quad(u \in K, g \in G, t \in T) .
$$

If $g \notin S$, then $a \notin \mathrm{gp}\left(S, T, \delta_{k} H\right)$. So we may concentrate on the case when $g \in S$. Suppose if possible that

$$
a \in \operatorname{gp}\left(S, T, \delta_{l} H\right) \text { for all } l \geqq k .
$$

Then $u \in \operatorname{gp}\left(S, T, \delta_{l} H\right)$ for every $l \geqq k$. Hence

$$
u \in[S, T] \delta_{l} H \quad(\text { for every } l \geqq k),
$$

where, as is customary

$$
[S, T]=\operatorname{gp}([s, t] \mid s \in S, t \in T) .
$$

But $[S, T]$ is a free factor of $K$ (see, e.g., K. W. Gruenberg [6]). Now $u \notin[S, T]$ a free factor of $K$. So $u \notin[S, T] \delta_{l} K$ for some $l \geqq 1$ (Lemma 3.1). This contradiction completes the proof of Proposition 2.

3.3. In order to be able to make use of Proposition 1 we will frequently call on the following theorem of F. Levin [14] which we formulate here as

LEMMA 3.2. Let $A$ be any group and let

$$
w\left(x, a_{1}, \ldots, a_{n}\right)=1 \quad\left(a_{1}, \ldots, a_{n} \in A\right)
$$

be an equation over $A$ in a single variable $x$. If there are no negative powers of $x$ occurring in $w\left(x, a_{1}, \ldots, a_{n}\right)$ then it is possible to solve the equation (1) in some supergroup $A^{*}$ of $A$. Moreover, if $A$ is solvable, then $A^{*}$ may also be chosen to be solvable of the same derived length as $A$.

COROLlaRY 3.2.1. Let $H$ be a group, let $X$ be a free group freely generated by the elements $x_{i}(i \in I)$ and let $F$ be the free product of $H$ and $X$. Let, further, $W=\left\{w_{\lambda} \mid \lambda \in \Lambda\right\}$ be a subset of $F$ such that

(i) the reduced form of each $w_{\lambda}$ contains only positive powers of the generators $x_{i}$ of $X$; 
(ii) each $w_{\lambda}$ contains at least one $x$ in its reduced form; and

(iii) if $\lambda \notin\left\{\mu_{1}, \ldots, \mu_{n}\right\}$ then $w_{\lambda}$ contains in its reduced form at least one $x$ which does not appear in any of $w_{\mu_{1}}, \ldots, w_{\mu_{n}}$. If

$$
R=\mathrm{gp}_{F}(W), \quad G=F / R \text { and } I=H R / R,
$$

then, for $i=1,2, \ldots$,

$$
H \cap\left(\delta_{i} F\right) R=\delta_{i} H \text { or, equivalently, } I \cap \delta_{i} G=\delta_{i} I .
$$

The proof of Corollary 3.2.1 is a straightforward application of Lemma 3.2. To see why, let $i$ be any positive integer and put $\bar{H}=H / \delta_{i} H$. Consider now the "equations" $\left\{\bar{w}_{\lambda}=1(\lambda \in \Lambda)\right\}$ where $\bar{w}_{\lambda}$ is obtained from $w_{\lambda}$ by replacing each element of $H$ by its image in $\bar{H}$. By Lemma 3.2 this system of equations can be simultaneously solved in a suitable supergroup $\bar{F}$ of $\bar{H}$ which is solvable of the same derived length as $\bar{H}$ as follows. First well-order $\Lambda$. If $\lambda$ is the first element in $\Lambda$, we can write $\bar{w}_{\lambda}$ in the form

$$
\bar{w}_{\lambda}=\bar{h}_{1} x_{i_{1}} \bar{h}_{2} x_{i_{2}} \cdots \bar{h}_{k} x_{i_{k}},
$$

where the $\bar{h}_{t} \in \bar{H}$ (and may be 1 ).

By Lemma 3.2 we can find a solvable supergroup $S_{1}$ of $\bar{H}$, of the same derived length, say $d$, as $\bar{H}$ which contains an element $a_{\lambda}$ such that

$$
\bar{h}_{1} a_{\lambda} \bar{h}_{2} a_{\lambda} \cdots \bar{h}_{k} a_{\lambda}=1 \text {. }
$$

Thus $x_{i_{t}}=a_{\lambda}(t=1,2, \ldots, k)$ is a solution of $\bar{w}_{\lambda}=1$ in $S_{1}$. This solution of $\bar{w}_{\lambda}=1$ enables us to find a simultaneous solution of $\bar{w}_{\lambda}=1$ and $\bar{w}_{\mu}=1$, where $\mu$ is the second (in the given well-order of $\Lambda$ ) element of $\Lambda$, in a solvable supergroup $S_{2}$ of $S_{1}$, which is also of derived length $d$. For let $\tilde{w}_{\mu}$ be the result of substituting $a_{\lambda}$ for each of the "variables" occurring both in $\bar{w}_{\mu}$ and $\bar{w}_{\lambda}$. By hypothesis there is at least one free variable in $\tilde{w}_{\mu}$. Hence, by Lemma 3.2, the equation $\tilde{w}_{\mu}=1$ over $S_{1}$ has a solution in a supergroup $S_{2}$ of $S_{1}$ which is solvable of derived length $d$. An easy transfinite induction patterned on the remarks above yields a solvable supergroup $\bar{F}$ of $\bar{H}$ of derived length $d$ which contains elements $a_{i}(i \in I)$ such that $x_{i}=a_{i}(i \in I)$ is a simultaneous solution of the equations $\bar{w}_{\lambda}=1$ over $\bar{H}$.

Now let $\eta$ be the natural homomorphism of $H$ onto $\bar{H}$ and let $\chi$ be the homomorphism of $X$ into $\bar{F}$ defined by

$$
x: x_{i} \rightarrow a_{i} \quad(i \in I) .
$$

Then $\eta$ and $\chi$ can simultaneously be extended to a homomorphism $\varphi$ of $F$ into $\bar{F}$. Let $K$ be the kernel of $\varphi$. Then $K \geqq \delta_{d} F$ since $\bar{F}$ is solvable of derived length $d$. Moreover, $K \geqq R$ since $x_{i}=a_{\imath}(i \in I)$ is a solution of the sytem of equations $\bar{w}_{\lambda}=1$. But $K \cap H=\delta_{i} H$ and since $d \leqq i,\left(\delta_{i} F\right) R \cap H=\delta_{i} H$, as desired.

4. The proof of Theorem 1.

4.1. Let

$$
G=(a, b, \ldots, c ; r)
$$


be a positive one-relator group where the relator $r$ is cyclically reduced. Our aim is to prove that $G \in R S$ where $S$ denotes the class of all solvable groups. The proof will involve a double induction on the length $|r|=n$ of the defining relator $r$. First, observe that if $n=1$, there is nothing to prove. We may assume, therefore, that $n>1$. Furthermore, inductively, we may suppose that if

$$
P=(u, v, \ldots, w ; s)
$$

is a positive one-relator group where the relator $s$ involves all of the generators and is cyclically reduced and if $|s|<n$, then

(1) if $Y$ is any proper subset of the generators of $P$, and if $a \in P, a \notin \mathrm{gp}(Y)$, then there is an integer $l$ such that $a \notin \delta_{l} P \cdot \mathrm{gp}(Y)$;

(2) $P \in R S$.

It should be noted that (2) is really a special case of (1), i.e., the case $Y=\varnothing$. However, it turns out to be convenient here to separate the two assumptions. First we shall prove, by making use of the assumptions (1) and (2), that $G \in R S$. Then we shall complete the proof by verifying that (1) holds in $G$.

4.2. Every one-relator group is the free product of a free group and a onerelator group whose defining relator involves all of the generators. Now free groups are residually solvable (F. W. Levi [13]) and the free product of residually solvable groups is in $R S(\mathrm{~K} . \mathrm{W}$. Gruenberg [6]). It follows from these remarks and Proposition 2 that we may assume that the defining relator $r$ of $G$ involves all of the generators $a, b, \ldots, c$.

If $G=\operatorname{gp}(a)$, there is nothing to prove. Thus we suppose $G$ comes equipped with at least two generators, $a$ and $b$. Suppose $a$ occurs with exponent sum $\alpha$ in $r$ and $b$ with exponent $\operatorname{sum} \beta$. We freely adjoin to $G$ a $\beta$ th root of $a$ by forming the generalized free product $\tilde{G}$ of $G$ and the infinite cyclic group $(d)$ on $d$ identifying $a$ with $d^{\beta}$ :

$$
\widetilde{G}=\left\{G *(d) ; a=d^{B}\right\}
$$

Putting $e=b d^{\alpha}$, we see that $\tilde{G}$ can be presented in the form

$$
\tilde{G}=\left(d, e, \ldots, c ; r\left(d^{\beta}, e d^{-\alpha}, \ldots, c\right)\right)
$$

where $r=r(a, b, \ldots, c)$ is the given defining relator of $G$. Of course, $d$ occurs with exponent sum zero in $\tilde{r}(d, e, \ldots, c)=r\left(d^{\beta}, e d^{-\alpha}, \ldots, c\right)$. Put

$$
\tilde{N}=\operatorname{gp}_{\tilde{G}}(e, \ldots, c) \text {. }
$$

Notice that the basic relator $\tilde{r}_{0}$ in $\tilde{N}$ (cf. 2.2) is positive and of lesser length than $r$. We use this fact to deduce that $\tilde{G} \in R S$.

4.3. Our first main objective in the proof of Theorem 1 is to show that $\tilde{G} \in R S$. Since $\tilde{G} / \tilde{N}$ is cyclic it is enough to prove $\tilde{N} \in R S$. The basis for the proof of this fact is the inductive hypothesis described in 4.1. Observe that (inductively) $N_{j} \in R S$ where (cf. 2.2)

$$
\begin{aligned}
N_{j}=\operatorname{gp}\left(e_{m(e)+j}, \ldots, e_{M(e)+j}, \ldots, c_{m(c)+j}, \ldots, c_{M(c)+j}\right. \\
\left.\quad r_{0}\left(e_{m(e)+j}, \ldots, e_{M(e)+j}, \ldots, c_{m(c)+j}, \ldots, c_{M(c)+j}\right)\right)
\end{aligned}
$$


$(j=0, \pm 1, \ldots)$. We take this opportunity to record that $N_{i, j}=\operatorname{gp}\left(N_{i}, N_{i+1}, \ldots, N_{j}\right)$ for every $i \leqq j$,

$$
N_{i, j+1}=\left\{N_{i, j} * N_{j+1} ; H_{j}\right\}
$$

and

$$
H_{j}=\mathrm{gp}\left(e_{m(e)+j+1}, \ldots, e_{M(e)+j}, \ldots, c_{m(c)+j+1}, \ldots, c_{M(c)+j}\right) .
$$

In order to prove $\tilde{N} \in R S$ we first prove that each of the subgroups $N_{i, j} \in R S$. This latter result is proved via the procedure called for by Proposition 1. To this end we prove now

Lemma 4.1. For every $i \geqq 1$ and every $j \geqq 1, \delta_{i} N_{1, j} \cap H_{0}=\delta_{i} H_{0}, \delta_{i} N_{1, j} \cap H_{j}$ $=\delta_{i} H_{j}$.

Lemma 4.1 follows from Corollary 3.2.1 on putting $H=H_{j}$, taking $X$ to be the free group on those generators which do not occur in $H$ and choosing $W$ to consist of the relators

$$
\tilde{r}_{0}\left(e_{m(e)+t}, \ldots, e_{M(e)+t} ; \ldots ; c_{m(c)+t}, \ldots, c_{M(c)+t}\right) \quad \text { with } t=1, \ldots, j .
$$

We emphasize that the generators, excluding those used to define $H$, are to be regarded as variables. Corollary 3.2.1 then yields

$$
\delta_{i} N_{1, j} \cap H_{j}=\delta_{i} H_{j}
$$

A similar argument shows that

$$
\delta_{i} N_{1, j} \cap H_{0}=\delta_{i} H_{0} .
$$

This completes the proof of Lemma 4.1.

Now it follows from Proposition 2 and the inductive assumption (1) (in 4.1) that $H_{j}$ is solvably separable in $N_{j+1}$. Let us assume also that $H_{j}$ is solvably separable in $N_{1, j}$. We shall prove that $N_{1, j+1}$ is $R S$ and that $H_{j+1}$ is solvably separable in $N_{1, j+1}$ (thereby justifying the added assumption that $H_{j}$ is solvably separable in $N_{1, j}$ ). As we remarked at the outset we shall make use of Proposition 1.

Observe that $N_{1, j+1}=\left\{N_{1, j} * N_{j+1} ; H_{j}\right\}$. By Lemma 4.1,

$$
\left(\delta_{i} N_{1, j}\right) \cap H_{j}=\delta_{i} H_{j}=\left(\delta_{i} N_{j+1}\right) \cap H_{j}
$$

for every $i$. Moreover, $H_{j}$ is solvably separable in both $N_{1, j}$ and $N_{j+1}$. So in order to be able to assert that $N_{1, j+1}$ is ${ }_{R} S$ we need only prove that

$$
P=\left\{N_{1, j} / \delta_{i} N_{1, j} * N_{j+1} / \delta_{i} N_{j+1} ; H_{j} \delta_{i} N_{1, j} / N_{1, j}=H_{j} \delta_{i} N_{j+1} / \delta_{i} N_{j+1}\right\}
$$

is $R S$ for every $i$. Put

$$
J=N_{1, j} / \delta_{i} N_{1, j}
$$

Let $\nu$ be the natural homomorphism of $N_{1, j}$ onto $J$ and let $w$ be the word obtained from $\tilde{r}_{0}\left(e_{m(e)+j+1}, \ldots, e_{M(e)+j+1} ; \ldots ; c_{m(c)+j+1}, \ldots, c_{M(c)+j+1}\right)$ by replacing each 
generator $e_{m(e)+j+1}, \ldots, e_{M(e)+j} ; \ldots ; c_{m(c)+j+1}, \ldots, c_{M(c)+j}$ by its image under $v$ in $J$. Then $w=1$ may be regarded as an equation over $J$. Hence by Lemma 3.2 it has a solution in a solvable supergroup $\bar{J}$ of $J$ of the same derived length $i$ as $J$ (see the proof of Corollary 3.2.1). Therefore, the identity mapping of $J$ onto $J$ can be extended to a homomorphism $\varphi_{1}$ of $P$ into $\bar{J}$. If $K_{1}$ is the kernel of $\varphi_{1}$, then

$$
K_{1} \cap J=1 .
$$

Similarly, we can find a normal subgroup $K_{2}$ of $P$ such that

$$
K_{2} \cap\left(N_{j+1} / \delta_{i} N_{j+1}\right)=1 .
$$

Hence, on putting $K=K_{1} \cap K_{2}$, we find

$$
K \cap J=1=K \cap\left(N_{j+1} / \delta_{i} N_{j+1}\right) .
$$

So by a theorem of Hanna Neumann [20], $K$ is free. But $P / K$ is solvable (of derived length $i$ ). Hence, remembering that free groups are ${ }_{R} S, P \in{ }_{R} S$. Thus we have proved part of the

LeMmA 4.2. $N_{1, j+1} \in R S$ for every $j \geqq 0$.

As we remarked above, we must prove also that $H_{j+1}$ is solvably separable in $N_{1, j+1}$ in order to validate Lemma 4.2. Let then $a \in N_{1, j+1}, a \notin H_{j+1}$. Now $a=a_{1} \cdots a_{t}$ where $a_{l} \in N_{1, j} \cup N_{j+1}(l=1, \ldots, t)$ and $a_{l}, a_{l+1}$ belong to different factors for every $l=1, \ldots, t-1$ (i.e., if $a_{l} \in N_{1, j}, a_{l+1} \notin N_{1, j}$ and similarly if $\left.a_{l} \in N_{j+1}\right)$. We consider two cases.

Case 1. $t=1$. This means either $a \in N_{1, j}$ or else $a \in N_{j+1}$. In the former case we know from the inductive assumptions that for a suitable choice of $i, a \notin\left(\delta_{i} N_{1, j}\right) H_{j}$. Putting $J=N_{1, j} / \delta_{i} N_{1, j}$ we can find a solvable supergroup $\bar{J}$ of $J$ of derived length $i$ and a homomorphism $\varphi_{1}$ of $N_{1, j+1}$ into $\bar{J}$ which maps $N_{1, j}$ onto $J$ according to the natural homomorphism of $N_{1, j}$ onto $J$ (see the remarks following the proof of Lemma 4.1). Therefore, $a \varphi_{1} \notin H_{j+1} \varphi_{1}$. Since $\bar{J}$ is solvable, $a \notin H_{j+1}\left(\delta_{i} N_{1, j+1}\right)$. We have still to consider the case where $a \in N_{j+1}$. An analogous argument centering around $N_{j+1} / \delta_{i} N_{j+1}$ (which again uses Lemma 3.2) yields the desired result.

Case 2. $t>1$. It follows here that we can find an $i$ such that the natural homomorphism of $N_{1, j}$ onto $N_{1, j} / \delta_{i} N_{1, j}$ and the natural homomorphism of $N_{j+1}$ onto $N_{j+1} / \delta_{i} N_{j+1}$ extends to a homomorphism $\varphi$, say of $N_{1, j+1}$ onto

$$
P=\left\{N_{1, j} / \delta_{i} N_{1, j} * N_{j+1} / \delta_{i} N_{j+1} ; H_{j} \delta_{i} N_{1, j} / \delta_{i} N_{1, j}=H_{j} \delta_{i} N_{j+1} / \delta_{i} N_{j+1}\right\},
$$

in such a way that $a \varphi \notin H_{j+1} \varphi$. But now we can (as before) choose a normal subgroup $K$ of $P$ satisfying (1) above where again $J=N_{1, j} / \delta_{i} N_{1, j}$. Notice that $K \cap H_{j+1} \varphi=1$. Moreover, $K$ is free. Suppose $a \varphi \in\left(H_{j+1} \varphi\right) K$. Then $a \varphi$ can be uniquely written in the form

$$
a \varphi=u v \quad\left(u \in H_{j+1} \varphi, v \in K\right) .
$$


Since $a \varphi \notin H_{j+1} \varphi, v \neq 1$. Choose $l$ sufficiently large to ensure that $v \notin \delta_{l} K$. It follows then that $a \varphi \notin\left(H_{j+1} \varphi\right)\left(\delta_{l} K\right)$. Therefore, $a \notin H_{j+1} \delta_{i+l} N_{1, j+1}$. This completes the proof of Case 2 and with it the proof of Lemma 4.2.

Finally we remark that

LEMMA 4.3. If $j \leqq j^{\prime}$ then

$$
\left(\delta_{i} N_{1, j^{\prime}}\right) \cap N_{1, j}=\delta_{i} N_{1, j}
$$

for every $i \geqq 1$.

The proof is a straightforward adaptation of the proof of Lemma 4.1 and will be omitted here.

We are now in a position to prove that $\tilde{N} \in R S$. Now every $N_{j, k} \in{ }_{R} S$ by Lemma 4.2. Moreover, by Lemma 4.3,

$$
\begin{aligned}
\delta_{i} \tilde{N} \cap N_{j, k} & =\delta_{i}\left(\bigcup_{r<0 ; s>0} N_{j+r, k+s}\right) \cap N_{j, k} \\
& =\left(\bigcup_{r<0 ; s>0} \delta_{i} N_{j+r, k+s}\right) \cap N_{j, k} \\
& =\bigcup_{r<0 ; s>0}\left(\left(\delta_{i} N_{j+r, k+s}\right) \cap N_{j, k}\right) \\
& =\bigcup_{r<0 ; s>0} \delta_{i} N_{j, k}=\delta_{i} N_{j, k} .
\end{aligned}
$$

It follows readily that $\tilde{N} \in R S$, as claimed.

4.4. We come now to the second part of the proof of Theorem 1. To this end let us consider again a positive one-relator group $G=(a, b, \ldots, c ; r)$. Let $Y$ be a proper subset of the given set of generators of $G$. Let $B=\operatorname{gp}(Y)$. We have to prove that if $g \in G, g \notin B$, then there is an $l$ such that $g \notin \delta_{l} G \cdot B$. We may assume that $Y \neq \varnothing$-this is the content of 4.3. Furthermore, we assume that $r$ is cyclically reduced and that $G$ comes equipped with at least two generators. Finally, we may assume $a \notin Y, b \in Y$.

Suppose now that $a$ occurs with exponent sum $\alpha \neq 0$ and that $b$ occurs with exponent $\operatorname{sum} \beta$. As in 4.2, we freely adjoin an $\alpha$ th root $y$ to $b$

$$
\tilde{G}=\left\{G *(y) ; y^{\alpha}=b\right\}
$$

Put $a=x y^{-\beta}$. Then $\tilde{G}$ is a group with a single defining relation:

$$
\tilde{G}=\left(x, y, \ldots, c ; r\left(x y^{-\beta}, y^{\alpha}, \ldots, c\right)\right) \text {. }
$$

Clearly $y$ occurs in $r$ with exponent sum zero. Now put $\widetilde{B}=\operatorname{gp}(Y, y)$. Clearly $g \notin \tilde{B}$. We shall prove

$$
g \notin \delta_{l} \tilde{G} \cdot \widetilde{B}
$$

for some $l$ which, of course, yields $g \notin \delta_{l} G \cdot B$ as desired.

Consider now $\tilde{N}=\operatorname{gp}_{G}(x, \ldots, c)$, where here the generator $y$ has been excluded from $\{x, \ldots, c\} . \tilde{N}$ is built up as described in $\S 2$. It is enough to prove (1) when 
$g \in \tilde{N}$. Notice again that $\tilde{N}$ is generated by the groups $N_{i}$ as described previously. The defining relators of the $N_{i}$ have smaller length than $r$ and so we inductively may assume that if $g \in N_{0}$, then there exists an $l$ such that $g \notin \delta_{l} N_{0} \cdot B_{0}$ where $B_{i}$ is the subgroup generated by the conjugates of the elements of $Y \backslash b$ by those powers of $y$ such that the resultant subscripts lie in the range of subscripts which go into the definition of $N_{i}$. It follows as in 4.3 that there is a homomorphism $v$ of $\tilde{N}$ into a solvable group such that $g \nu \notin \widetilde{B} \nu$ and so the result follows.

We are left with the case where $g \notin N_{0}$. In order to take care of this case we must interrupt our discussion with the following proposition.

Proposition 3. Suppose

$$
A=\operatorname{gp}\left(a_{1}, \ldots, a_{m}\right), \quad B=\operatorname{gp}\left(b_{1}, \ldots, b_{n}\right)
$$

are finitely generated groups with isomorphic subgroups

$$
H=\operatorname{gp}\left(a_{1}, \ldots, a_{r}\right), \quad K=\operatorname{gp}\left(b_{1}, \ldots, b_{r}\right)
$$

with $a_{i} \rightarrow b_{i}(i=1, \ldots, r)$ defining an isomorphism from $H$ to $K$. Let $G$ be the generalized free product of $A$ and $B$ amalgamating $H$ with $K$ according to the given isomorphism:

$$
G=\left\{A * B ; a_{1}=b_{1}, \ldots, a_{r}=b_{r}\right\}
$$

Furthermore, let

$$
F=\operatorname{gp}\left(a_{i_{1}}, \ldots, a_{i_{k}}, b_{i_{1}}, \ldots, b_{i_{k}}\right) .
$$

Finally let $\varphi$ be a homomorphism of $G$ into a group $G^{*}$ such that

(i) $\varphi \mid A$ is a monomorphism;

(ii) $\varphi \mid B$ is a monomorphism;

(iii) $a_{i_{1}} \varphi=b_{i_{1}} \varphi, \ldots, a_{i_{k}} \varphi=b_{i_{k}} \varphi$.

If $K$ is the kernel of $\varphi$ then $K$ is free and $K \cap F$ is a free factor of $K$.

In order to prove Proposition 3, we need to recall some work of Karrass and Solitar (see [17]) concerning groups given by generators and defining relations. For convenience we shall formulate whatever definitions and results we need in terms of the group $G$ above with its subgroup $K$ the object of the discussion.

We term the generators $a_{1}, \ldots, a_{m} \alpha$-generators and the generators $b_{1}, \ldots, b_{n}$ $\beta$-generators. Choose two representative functions $\alpha$ and $\beta$ of the cosets of $K$ in $G$. We denote the " $\alpha$-representative" of the element $w \in G$ by $w^{\alpha}$ and similarly for the $\beta$-representative of $w$. The functions $\alpha$ and $\beta$ are termed extended Schreier representative functions if for each representative $N$ (this means that either $N=w^{\alpha}$ or $N=w^{\beta}$ for some $w \in G)$ of the form $N \equiv M x^{\varepsilon}(\varepsilon= \pm 1)$ both $N$ and $M$ are $\delta$-representatives if $x$ is a $\delta$-generator, $\delta \in\{\alpha, \beta\}$. Extended Schreier representative functions are termed regular if the words obtained by removing the $\delta$-generators from the ends of the $\delta$-representatives comprise a system of representatives for the double $(K, D)$ - 
cosets in $G$ where $D$ is the subgroup generated by the $\delta$-generators, $\delta \in\{\alpha, \beta\}$. For each $\beta$-representative $N$, define

$$
t_{N}=N\left(N^{\alpha}\right)^{-1} .
$$

It then transpires that $K$ is generated by these generators $t_{N}$ ( $N$ any $\beta$-representative) subject to the defining relations $t_{N}=1$ if $N$ is freely equal to $N^{\alpha}$ and, corresponding to each $\alpha$-representative $M$ and each integer $i=1,2, \ldots, r$, the further relations $t_{\left(M a_{i}\right)^{\beta}}=t_{M^{\beta}}$.

We are now in a position to prove Proposition 3. Let us choose the regular extended Schreier representative functions $\alpha$ and $\beta$. This can be done in such a way that if a coset $K g$ contains an element of $F$ then the $\alpha$-representative is a word in $a_{i_{1}}, \ldots, a_{i_{k}}$ and the $\beta$-representative is a word in $b_{i_{1}}, \ldots, b_{i_{k}}$.

Consider next the restriction $\varphi^{*}$ of $\varphi$ to $F$. Now $F$ is itself a free product with an amalgamated subgroup. Therefore, the above remarks are valid also for $F$. Indeed the respective restrictions $\alpha^{*}$ and $\beta^{*}$ of $\alpha$ and $\beta$ to $F$ are regular extended Schreier representative systems for the kernel $K^{*}$ of $\varphi^{*}$ in $F$. Therefore, $K^{*}$ is generated by the generators $t_{N}$ where $N$ ranges over the $\beta^{*}$-representatives. The defining relations of $K^{*}$ in terms of these generators are simply

$$
t_{N}=1 \text { if } N \text { is freely equal to } N^{\alpha}
$$

and

$$
t_{\left(M a_{1}\right)^{\beta^{\beta}}}=t_{M^{\beta}} \quad(1 \leqq j \leqq k)
$$

where here $N$ ranges over all $\beta^{*}$-representatives and $M$ ranges over all $\alpha^{*}$ representatives.

Now $K \cap F=K^{*}$. So it follows that $K \cap F$ is the subgroup of $F$ generated by those $t_{N}$ for which $N$ is a $\beta^{*}$-representative. The proof of Proposition 3 now follows from the following obvious

LEMMA 4.4. Let I be a nonempty index set, $J$ a subset of $I$ and $\Delta$ a subset of $I \times I$. Suppose that $K$ is a group with the presentation

$$
K=\left(t_{i}(i \in I) \mid t_{i}=t_{j} \text { if }(i, j) \in \Delta, t_{j}=1 \text { if } j \in J\right) .
$$

Let $I^{*}$ be $a$ subset of $I$ and let

$$
K^{*}=\operatorname{gp}\left(t_{i} \mid i \in I^{*}\right) .
$$

Then $K$ is free and $K^{*}$ is a free factor of $K$.

The key fact we need in order to be able to complete the proof of Theorem 1 is the following lemma (we assume here the notation of Proposition 3).

Lemma 4.5. If $g \in G, g \notin F$ and $G^{*}$ is solvable, then there exists an $l$ such that $g \notin \delta_{l} G \cdot F$. 
Proof. Suppose $g \notin K F$. Then the result follows easily since $G^{*}$ is solvable and so $\delta_{l} G \leqq K$ for a suitable choice of $l$.

Suppose then that $g \in K F$. So for some $f \in F, g f^{-1} \in K$. But $K \cap F$ is a free factor of $K$ :

$$
K=(K \cap F) * S .
$$

Now $g f^{-1} \notin K \cap F$ and $K$ is free and hence residually solvable. Therefore, by Lemma 3.1., $K \cap F$ is solvably separable in $K$. Hence

$$
g f^{-1} \notin\left(\delta_{l} K\right) \cdot(K \cap F)
$$

for a suitable choice of $l$. This means that $g f^{-1} \notin\left(\delta_{l} K\right) \cdot F$, as desired. For suppose the contrary. Then $g f^{-1}=u v\left(u \in \delta_{l} K, v \in F\right)$. This means that $u^{-1} g f^{-1} \in K \cap F$ and hence that $g f^{-1} \in(K \cap F) \cdot\left(\delta_{l} K\right)$, a contradiction. So the proof of Lemma 4.5 is complete.

4.5. We are now able to complete the proof of Theorem 1 . For $i \leqq j$ we define $B_{i, j}=\operatorname{gp}\left(B_{i}, \ldots, B_{j}\right)$. Inductively we may assume that if $f \in N_{i, j}, f \notin B_{i, j}$, then there exists an $l$ such that $f \notin\left(\delta_{l} N_{i, j}\right) \cdot B_{i, j}(i \leqq j)$. Suppose now that $g \in N_{i, j+1}$. Then, remembering the choice of $g$ at the outset (cf. the beginning of 4.4), we have $g=x_{1} \cdots \cdots x_{n}$ where the $x_{i}$ come alternately from $N_{i, j}$ and $N_{j+1}$ and either at least one of the $x_{i}$ lies outside $B_{i, j}$ for some $x_{i} \in N_{i, j}$ or else some $x_{i}$ in $N_{j+1}$ lies outside $B_{j+1}$.

Now by Lemma 4.1 it follows that

$$
\left(\delta_{l} N_{i, j}\right) \cap H_{j}=\left(\delta_{l} N_{j+1}\right) \cap H_{j}=\delta_{l} H_{j} .
$$

So we can again form the generalised free product $\bar{N}_{i, j+1}$ of $N_{i, j} / \delta_{l} N_{i, j}$ and $N_{j+1} / \delta_{l} N_{j+1}$ amalgamating $H_{j} / \delta_{l} H_{j}$ :

$$
\bar{N}_{i, j+1}=\left\{N_{i, j} / \delta_{l} N_{i, j} * N_{j+1} / \delta_{l} N_{j+1} ; H_{j} / \delta_{l} H_{j}\right\} .
$$

It follows from the inductive hypothesis that if $l$ is chosen sufficiently large then the natural image $\bar{g}$ of $g$ in $\bar{N}_{i, j+1}$ lies outside the image $\bar{B}_{i, j+1}$ of $B_{i, j+1}$. By Lemma 4.5 we can therefore conclude that for a suitably large choice of $k \geqq l$ we have

$$
\bar{g} \notin\left(\delta_{k} \bar{N}_{i, j+1}\right) \bar{B}_{i, j+1} \text {. }
$$

But a repeated appeal to Lemma 4.3 yields $g \notin\left(\delta_{k} N\right) B_{\infty, \infty}$ where

$$
B_{\infty, \infty}=\operatorname{gp}\left(B_{i, j} \mid i, j \text { any integers, } i \leqq j\right) .
$$

So $g \notin\left(\delta_{k+1} \tilde{G}\right) B$ as desired. This completes the proof of Theorem 1 .

\section{The proof of Theorem 2.}

5.1. We will find it useful to express Theorem 2 in slightly different terms than those used in the introduction. To this end we define, for any group $G$,

$$
\sigma_{1} G=\bigcap_{n=1}^{\infty} \lambda_{n} G
$$


and, inductively,

$$
\sigma_{i+1} G=\sigma_{1}\left(\sigma_{i} G\right) \quad(i \geqq 1),
$$

where $\lambda_{n} G$ is itself defined by $\lambda_{n} G / \lambda_{n} G=$ the torsion subgroup of $G / \lambda_{n} G$ and $\lambda_{n} G$ is the $n$th term of the lower central series of $G$. We term the series

$$
G \geqq \sigma_{1} G \geqq \sigma_{2} G \geqq \cdots
$$

the lower $\sigma$-series of $G$. The first $d$ such that $\sigma_{d} G=\sigma_{d+1} G$ is termed the depth of $G$.

Theorem 2 may now be reformulated as follows (where as usual $L^{\prime}$ denotes the derived group of the group $L$ ).

THEOREM 2. The depth $d$ of a positive one-relator group $G$ is finite. If $G$ is torsionfree $\sigma_{d} G=1$. If $G$ contains an element $(\neq 1)$ of finite order then $\left(\sigma_{d} G\right)^{\prime}$ is free and $\sigma_{d} G /\left(\sigma_{d} G\right)^{\prime}$ is a direct sum of cyclic groups of the same finite order and a free abelian group.

5.2. The proof of Theorem 2 depends on the following three lemmas, the first of which is an analogue of Corollary 3.2.1.

LEMMA 5.1. Let $H$ be a free group, $J$ a second free group freely generated by the elements $x_{i}(i \in I)$ and let $F$ be the free product of $H$ and J. Let, further, $W=\left\{w_{\lambda} \mid \lambda \in \Lambda\right\}$ be a subset of $F$ such that

(i) the reduced form of each $w_{\lambda}$ contains only positive powers of the generators $x_{i}$ of $\mathrm{J}$;

(ii) each $w_{\lambda}$ contains at least one $x$ in its reduced form; and

(iii) if $\lambda \notin\left\{\mu_{1}, \ldots, \mu_{m}\right\}$, then $w_{\lambda}$ (in its reduced form) contains at least one $x$ which does not appear in any of $w_{\mu_{1}}, \ldots, w_{\mu_{m}}$.

If $R=\mathrm{gp}_{F}(W)$ then, for $n=1,2, \ldots, H \cap\left(\lambda_{n} F\right) R=\lambda_{n} H$.

Proof. Let $n$ be any chosen integer and suppose that $H$ is freely generated by $\left\{a_{\gamma} \mid \gamma \in \Gamma\right\}$. Let $\hat{F}$ be the free nilpotent $\mathscr{D}$-group (see [3]) of class $n-1$ freely generated by

$$
\left\{\hat{a}_{\gamma} \mid \gamma \in \Gamma\right\} \cup\left\{\hat{x}_{i} \mid i \in I\right\} .
$$

Now in the free nilpotent $\mathscr{D}$-group $\hat{F}, \hat{F} / \delta_{2} \hat{F}$ is additively, a vector space over the field of rational numbers. The elements in $\left\{\hat{a}_{\gamma} \mid \gamma \in \Gamma\right\} \cup \hat{W}$ are linearly independent modulo $\delta_{2} \hat{F}$ (where $\hat{W}$ is obtained from $W$ by replacing each $w_{\lambda}$ by $\hat{w}_{\lambda}$, the result of substituting $\hat{a}_{\gamma}$ for $a_{\gamma}$ and $\hat{x}_{i}$ for $x_{i}$ in $w_{\lambda}$ when appropriate)-this follows from the conditions (i), (ii) and (iii). Hence modulo $\delta_{2} \hat{F},\left\{\hat{a}_{\gamma} \mid \gamma \in \Gamma\right\} \cup \hat{W}$ can be enlarged to a basis $B$ for $\hat{F}$. But any set of elements of a nilpotent $\mathscr{D}$-group which generates modulo the derived group is a set of $\mathscr{D}$-generators. Moreover, any set of elements of a free nilpotent $\mathscr{D}$-group which is linearly independent modulo the derived group freely generates a free nilpotent $\mathscr{D}$-group (of the same class) (cf. [3]). It follows that $B$ freely generates $\hat{F}$. Hence, if $\hat{R}=\operatorname{gp}_{\hat{k}}(\hat{W}), \hat{F} / \hat{R}$ is a free nilpotent $\mathscr{D}$-group. Thus $\left\{\hat{a}_{\gamma} \hat{R} \mid \gamma \in \Gamma\right\}$ freely generates a free nilpotent $\mathscr{D}$-group of class $n-1$. 
So the subgroup (not the $\mathscr{D}$-group, but the usual subgroup) generated by $\left\{\hat{a}_{\gamma} \hat{R} \mid \gamma \in \Gamma\right\}$ is free nilpotent of class $n-1$ [3]. Now the mapping defined by

$$
a_{y} \rightarrow \hat{a}_{y} \hat{R}, \quad x_{i} \rightarrow \hat{x}_{i} \hat{R}
$$

determines a homomorphism $\varphi$ of $F$ into $\hat{F} / \hat{R}$. Clearly $R$ and $\lambda_{n} F$ are contained in the kernel $K$ of $\varphi$. Since it is clear from the preceding remarks that $K \cap H=\lambda_{n} H$, it follows that $\left(\lambda_{n} F\right) R \cap H=\lambda_{n} H$ as desired.

COROLLARY 5.1.1. Under the hypothesis of Lemma 5.1, if $\lambda_{n}(F / R)=T / R$ then $T \cap H=\lambda_{n} H$.

Corollary 5.1.1 follows immediately from the proof of Lemma 5.1.

We prove next

Lemma 5.2. Let $P$ be the generalised free product of its subgroups $A$ and $B$ amalgamating the subgroup $C$ :

$$
P=\{A * B ; C\}
$$

If $M$ is a normal subgroup of $P$ which intersects $C$ in the identity subgroup, then $M \cap A$ and $M \cap B$ are free factors of $M$.

The proof is an immediate consequence of Theorem 5 of [11].

Corollary 5.2.1. Let $G$ be $a$ one-relator group generated by $a, b, \ldots, c$ and defined in terms of these generators by the single relator $r$. Suppose a occurs with exponent sum zero in $r$ and that $N=\mathrm{gp}_{G}(b, \ldots, c)$. Let the subgroups $N_{i}$ and $H_{i}$ of $N$ be defined as in 2.2. Then every normal subgroup $M$ of $N$ which meets each of the subgroups $H_{i}$ trivially is a free product of conjugates of subgroups of the groups $\ldots, N_{-1}, N_{0}, N_{1}, \ldots$.

Proof. Put

$$
M_{2 i}=M \cap N_{-i, i}, \quad M_{2 i+1}=M \cap N_{-i, i+1} \quad(i \geqq 0) .
$$

Then $M=\bigcup_{i=0}^{\infty} M_{i}$. Now $M_{0}=M \cap N_{0}, M_{1}=M \cap N_{0,1}$. So, by Lemma 5.2, $M_{0}$ is a free factor of $M_{1}$. Notice that $M_{1}$ is a normal subgroup of $N_{0,1}$ which meets $H_{1}$ trivially. Hence $M_{1}$ is a free product of a free group and conjugates of subgroups of $N_{0}$ and $N_{1}$. Continuing in this way we find that $M_{i}$ is a free factor of $M_{i+1}$ and that $M_{i+1}$ is a free product of conjugates of subgroups of the various factors $N_{j}$ and a free group. Thus we may for each $i$ express $M_{i+1}$ in the form

$$
M_{i+1}=M_{i} * L_{i} \text {. }
$$

It follows that $M$ is the free product of its subgroups $M_{0}, L_{0}, L_{1}, \ldots$ :

$$
M=M_{0} * \prod_{i=0}^{\infty} * L_{i} .
$$

Hence $M$ has the desired structure. 
The third and final lemma we require is

LEMMA 5.3. Let $\left\langle A_{i} ; i \in I\right\rangle$ be a family of groups. Suppose $A_{i}$ is of finite depth $d_{i}$, and that $\left(\sigma_{d_{i}} A_{i}\right)^{\prime}$ is free $(i \in I)$. Suppose furthermore that the $d_{i}$ are bounded above and that $d$ is the least upper bound of the $d_{i}$. Let $A$ be the free product of the groups $A_{i}$ :

$$
A=\prod_{i \in I}^{*} A_{i} \text {. }
$$

Then $A$ is of depth $d$. Moreover, $\left(\sigma_{d} A\right)^{\prime}$ is free and if each $\sigma_{d_{i}} A_{i} /\left(\sigma_{d_{i}} A_{i}\right)^{\prime}$ is the direct sum of cyclic groups of the same finite order l, irrespective of $i \in I$, then $\sigma_{d} A /\left(\sigma_{d} A\right)^{\prime}$ is also a direct sum of finite cyclic groups all of the same finite order l and a free abelian group.

Proof. The free product of residually-torsion-free-nilpotent groups is residuallytorsion-free-nilpotent (A. I. Mal'cev [18]). Moreover it is not difficult to prove that $\sigma_{d} A \geqq \sigma_{d_{i}}\left(A_{i}\right)$ for all $i \in I$. Thus, by the remark above and the Kurosh subgroup theorem for free products, $\sigma_{d} A$ is the free product of conjugates of the subgroups $\sigma_{d_{i}} A_{i}(i \in I)$ and a free group. Now $\left(\sigma_{d_{i}} A_{i}\right)^{\prime}$ is free for every $i$. Thus it follows, again by the Kurosh subgroup theorem for free products, that $\left(\sigma_{d} A\right)^{\prime}$ is free and that $\sigma_{d} A /\left(\sigma_{d} A\right)^{\prime}$ has the desired form.

We are now in a position to prove Theorem 2. Thus let

$$
G=(a, b, \ldots, d ; r)
$$

be a positive one-relator group. By Lemma 5.3 we may assume that $r$ involves all of the given generators $a, b, \ldots, d$. The proof is by induction on the length $|r|$ of the defining relator $r$. If $|r|=1, G=1$ and there is nothing more to do. Thus we assume $|r|>1$ and proceed as in 4.2 to consider the group $\tilde{G}$ defined in 4.2 with its normal subgroup $N$ where $\tilde{G} / N$ is infinite cyclic. Now if we think of $N$ as being built up from its subgroups $N_{i}$ then we readily find that $\lambda_{\omega} N\left(=\bigcap_{n=1}^{\infty} \lambda_{n} N\right)$ meets each of the subgroups $H_{i}$ trivially (Corollary 5.1.1). So by Corollary 5.2.1 $\lambda_{\omega} N$ is a free product of conjugates of subgroups of the groups $N_{i}$. Now inductively each $N_{i}$ has a $\sigma$-series of the desired type. Hence by Lemma 5.3 the result follows. So the proof of Theorem 2 is complete.

5.3. We have actually proved that a torsion-free positive one-relator group is poly-residually-torsion-free-nilpotent. Moreover, each of the residually-torsionfree-nilpotent factors are residually finite $p$-groups for every prime $p$. This follows from our proof of Theorem 2, the fact that finitely generated torsion-free-nilpotent groups are residually finite $p$-groups for every prime $p$ and the fact that the free product of groups which are residually finite $p$-groups is again residually a finite p-group (K. W. Gruenberg [6]). The various corollaries of Theorem 2 follow from these remarks.

It remains only to verify that not every one-relator group is poly-residuallyfinite. Indeed let

$$
G=\left(a, b ; a=a^{-1} b^{-1} a^{-1} b a b^{-1} a b\right) .
$$


Then it follows from the argument in [4] that if $a$ is of finite order modulo some normal subgroup $L$ of $G$ then $a \in L$. Thus $G$ is not poly-residually-finite.

\section{The proof of Theorem 3.}

6.1. We are left only with the proof of

TheOREM 3. Let $A$ and $B$ be residually-torsion-free-nilpotent, let $a \in A, b \in B$ $(a \neq 1 \neq b)$ and $G$ be the generalized free product of $A$ and $B$ identifying $a$ with $b$ :

$$
G=\{A * B ; a=b\} .
$$

Then $G$ is an extension of a residually-torsion-free-nilpotent group by another residually-torsion-free-nilpotent group.

Proof. Since both $A$ and $B$ are residually-torsion-free-nilpotent we can find $m \geqq 1$ and $n \geqq 1$ such that $a \in \lambda_{m} A, a \notin \lambda_{m+1} A, b \in \lambda_{n} B, b \notin \lambda_{n+1} B$. Let $D$ be the central product of $A / \lambda_{m+1} A$ and $B / \lambda_{n+1} B$ amalgamating $a \lambda_{m+1} A$ with $b \lambda_{n+1} B$ (see e.g., A. G. Kurosh [12]):

$$
D=\left\{A / \lambda_{m+1} A \times B / \lambda_{n+1} B ; a \lambda_{m+1} A=b \lambda_{n+1} B\right\} .
$$

Let $\tilde{T}$ be the torsion-subgroup of $D$. Then

$$
A / \lambda_{m+1} A \cap \tilde{T}=1=B / \bar{\lambda}_{n+1} B \cap \tilde{T} .
$$

Let $T$ be the preimage of $\tilde{T}$ in $G$. Then

$$
T \cap \operatorname{gp}(a)=1=T \cap \mathrm{gp}(b) .
$$

So $T$ is a free product of conjugates of subgroups of $A$ and $B$ and a free group [20]. Hence $T$ is residually-torsion-free-nilpotent (A. I. Mal'cev [18]). Since $G / T$ is itself torsion-free-nilpotent, the proof of Theorem 3 is complete.

Thus, by Theorem 3, the group $G$ above is meta-ordered and so by a theorem of I. P. Hughes [10] its integral group ring can be embedded in a division ring.

\section{REFERENCES}

1. Benjamin Baumslag, Residually free groups, Proc. London Math. Soc. (3) 17 (1967), 402-418. MR 35 \#6738.

2. Gilbert Baumslag, On the residual finiteness of generalised free products of nilpotent groups, Trans. Amer. Math. Soc. 106 (1963), 193-209. MR 26 \#2489.

3. - Some remarks on nilpotent groups with roots, Proc. Amer. Math. Soc. 12 (1961), 262-267. MR 23 \#A934.

4. - A non-cyclic one-relator group all of whose finite quotients are cyclic, J. Austral. Math. Soc. 10 (1969), 497-498.

5. Gilbert Baumslag and Donald Solitar, Some two-generator one-relator non-Hopfian groups, Bull. Amer. Math. Soc. 68 (1962), 199-201. MR 26 \#204.

6. K. W. Gruenberg, Residual properties of infinite solvable groups, Proc. London Math. Soc. (3) 7 (1957), 29-62. MR 19, 386.

7. P. Hall, On the finiteness of certain soluble groups, Proc. London Math. Soc. (3) 9 (1959), 595-622. MR 22 \#1618. 
8. P. Hall, The Frattini subgroups of finitely generated groups, Proc. London Math. Soc. (3) 11 (1961), 327-352. MR 23 \#A1718.

9. Graham Higman, The units of group-rings, Proc. London Math. Soc. (2) 46 (1940), 231-248. MR 2, 5.

10. I. P. Hughes, Division rings of fractions for group rings, Comm. Pure Appl. Math. (to appear).

11. A. Karrass and D. Solitar, The subgroups of a free product of two groups with an amalgamated subgroup, Trans. Amer. Math. Soc. 144 (1970), 227-255.

12. A. G. Kuros, Theory of groups, GITTL, Moscow, 1953; English transl., vol. 2, Chelsea, New York, 1955. MR 15, 501; MR 17, 124.

13. F. W. Levi, Uber die Untergruppen der freien gruppen. II, Math. Z. 37 (1933), 90-97.

14. Frank Levin, Solutions of equations over groups, Bull. Amer. Math. Soc. 68 (1962), 603604. MR 26 \#212.

15. Wilhelm Magnus, Beziehungen zwischen gruppen und idealen in einem speziellen ring, Math. Ann. 111 (1935), 259-280.

16. _- Residually finite groups, Bull. Amer. Math. Soc. 75 (1969), 305-316. MR 39 \#2865.

17. W. Magnus, A. Karrass and D. Solitar, Combinatorial group theory: Presentations of groups in terms of generators and relations, Pure and Appl. Math., vol. 13, Interscience, New York, 1966. MR 34 \#7617.

18. A. I. Mal'cev, Generalised nilpotent algebras and their adjoint groups, Mat. Sb. 25 (67) (1949), 347-366; English transl., Amer. Math. Soc. Transl. (2) 69 (1968), 1-21. MR 11, 323.

19. - On the faithful representation of infinite groups by matrices, Mat. Sb. 8 (50) (1940), 405-422; English transl., Amer. Math. Soc. Transl. (2) 45 (1965), 1-18. MR 2, 216.

20. H. Neumann, Generalized free products with amalgamated subgroups. II, Amer. J. Math. 71 (1949), 491-540. MR 11, 8.

21. B. B. Newman, Some results on one-relator groups, Bull. Amer. Math. Soc. 74 (1968), 568-571. MR 36 \#5204.

22. H. Seifert and W. Threlfall, Lehrbuch der topologie, reprint, Chelsea, New York, 1947.

Rice UNIVERSITY,

Houston, Texas 77001 